\title{
BMJ Open Quality The game of telephone: a sustained, low-cost, quality improvement initiative to enhance communication between patients and their resident physician
}

\author{
Amanda Schnell, ${ }^{1}$ Sarah Stolte, ${ }^{1}$ Melissa Taylor, ${ }^{2}$ Jane Broxterman ${ }^{1}$
}

To cite: Schnell A, Stolte S, Taylor M, et al. The game of telephone: a sustained, lowcost, quality improvement initiative to enhance communication between patients and their resident physician.BMJ Open Quality 2017;6:e00143. doi:10.1136/ bmjoq-2017-000143

Received 15 June 2017 Revised 10 August 2017 Accepted 18 August 2017

\section{CrossMark}

${ }^{1}$ Department of General Medicine, The University of Kansas Health System, Kansas City, Kansas, USA

Internal Medicine, The University of Kansas Health System, Kansas City, Kansas, USA

Correspondence to Dr Jane Broxterman; jbroxterman@kumc.edu

\section{ABSTRACT}

This multidisciplinary quality improvement project was designed to enhance telephone communication between patients and their resident physician while concomitantly creating a standardised telephone communication protocol for resident internal medicine continuity clinics. The plan, do, study, act (PDSA) quality improvement framework model was applied for four distinct cycles. Baseline data were collected regarding open telephone encounters. The initial intervention entailed targeted communication to specific individual residents with open telephone encounters more than one SD above the average. The next cycle involved developing a novel communication process map that was distributed to faculty preceptors and clinic anchor nurses. The faculty preceptors then disseminated the new policies and communication algorithm to resident physicians. Finally, new resident and anchor nurses were educated about the standardised processes through scheduled orientation activities. After 19 months of implementation of this project with four PDSA cycles, resident open telephone encounters decreased by $40.7 \%$. Resident telephone communication in continuity clinics can be improved through targeted individualised communication, implementation of a standardised telephone communication protocol, dissemination of communication algorithms to clinic faculty, residents and nurses and ongoing education to all parties through orientation activities to instil a self-sustaining culture change.

\section{PROBLEN}

At our institution, our internal medicine resident continuity clinic operates on an ' $\mathrm{x}+\mathrm{y}$ ' rotational schedule, which separates traditional training blocks to dedicated inpatient/ consult rotations and dedicated continuity/ ambulatory rotational experiences. Our residents are divided into specific firm groups, which will have a 3-week sequence of non-ambulatory rotations, followed by a 1 week dedicated continuity clinic week. We have a strict empanelment process for resident continuity clinic, and even if a resident is not on a continuity clinic rotation, the resident is still responsible for the care of their patient panel. At our institution, each resident has an assigned faculty preceptor and anchor nurse. Prior to intervention, residents were responsible for checking an 'inbox' via the electronic medical record (EMR) on an implied, but not standardised frequent basis.

Before our quality improvement project, internal medicine residents had 270 open phone encounters, indicating poor communication practices.

Due to a complicated algorithm and no standardisation, each resident, faculty and anchor nurse team was handling the phone calls differently (figure 1). In the EMR inbox, patient phone calls are important measures of intraoffice visit communication and patient care. At our institution, unanswered phone calls are monitored bimonthly by an institutionally generated report. Our initial aim was to reduce active resident phone calls by $15 \%$ over 2 months. We created a diverse focus group made of ambulatory faculty, residents, anchor nurses and residency and nursing leadership to develop a standardised communication tool and protocol .

\section{BACKGROUND}

There has been little research focused on internal medicine resident physician telephone communication since the work of the Telephone Encounters Learning Initiative Group in the 1990s. Much has evolved since that time, including the implementation of widespread electronic health records, Accreditation Council for Graduate Medical Education (ACGME) regulations and a more connected patient population. In addition, managed care, advances in medical knowledge and an ageing population have all increased, compounding the challenges of telephone communication between office visits. ${ }^{1}$ Many newly practising physicians report the desire for additional education in this area. ${ }^{2-4}$ Standardised curriculum and algorithms in dealing with telephone medicine are not widely 


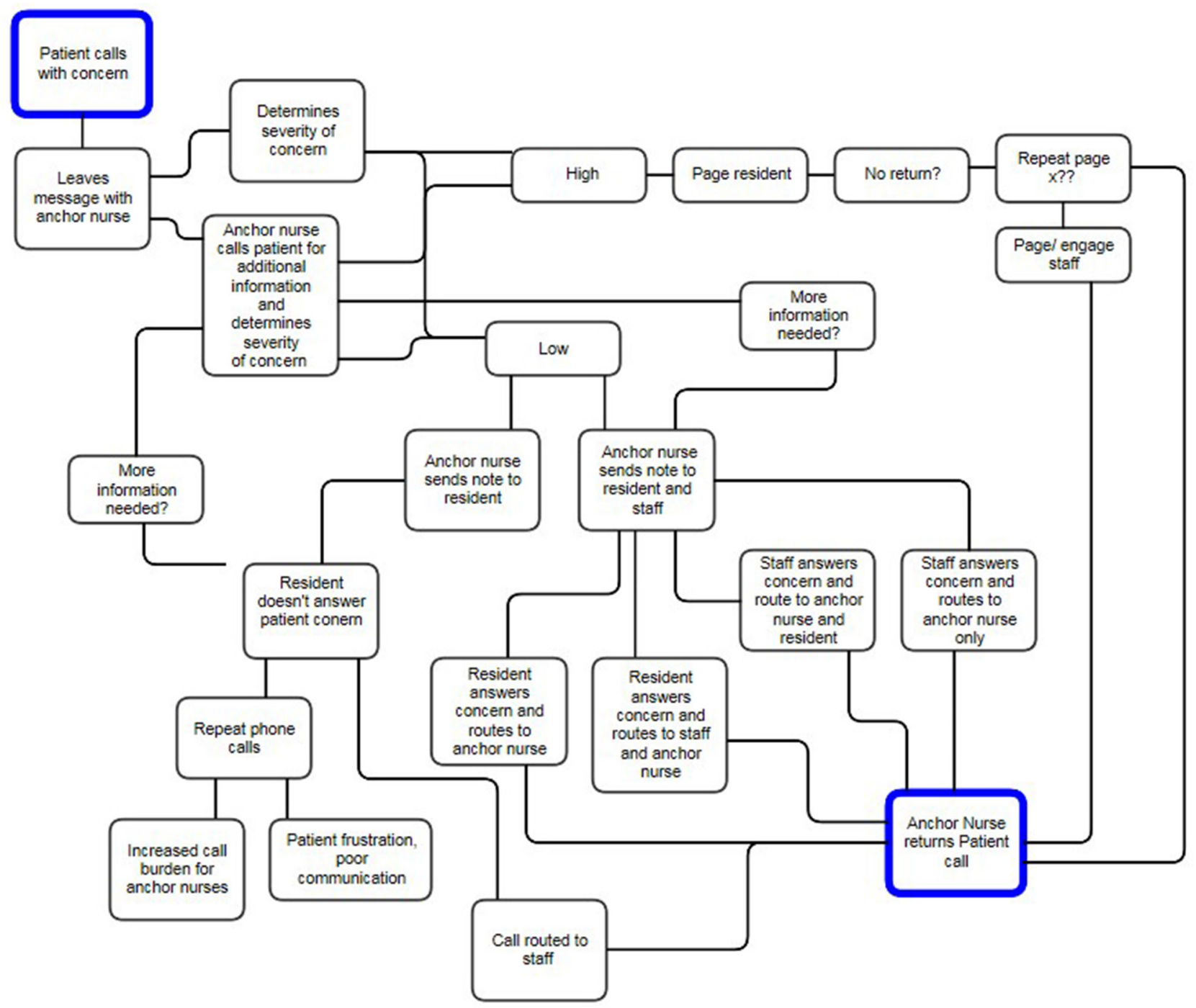

Figure 1 Original communication algorithm and protocol.

available or published. ${ }^{256}$ Non-standardised algorithms may lead to patient safety and quality issues. Communication failures including poor documentation, scheduling problems and miscommunication have been shown to play a central role in medical mishaps from inconvenience and anxiety to serious compromises in patient safety. ${ }^{78}$ In fact, telephone-related medical malpractice in the ambulatory setting is significant and costly. ${ }^{9}$

\section{MEASUREMENT}

For this intervention, data were collected from an institutionally generated bimonthly report. Data for the report are compiled from the Clarity database provided by Epic. These scheduled reports to the Business Objects Enterprise Server show point-in-time data on incomplete phone encounters of all resident physicians. This specific report was chosen because of accessibility and accuracy. Sixty-five resident physicians were included in data analysis. Baseline data showed 270 open phone encounters prior to intervention. To evaluate our project aim of decreasing open telephone encounters, we used data from these bimonthly enterprise reports delivered via email.

\section{DESIGN}

A simple communication algorithm and protocol was developed with input from a focus group which comprises faculty, residents, clinic anchor nurses and clinic nursing leadership (figure 2). Faculty and nursing attended required telephone communication training sessions. These sessions were incorporated into standard operational meeting times, which was at no added cost to the system. Education was given by respective leadership to enhance clinical work environment and communication. The algorithm was disseminated to all residents by faculty preceptors as well as programme leadership. Training included identification of clearly defined roles for each member of the care team regarding telephone management. A communication card was developed where residents identified their next rotation and accessibility. The anchor nurses kept the cards as they are the first line of 


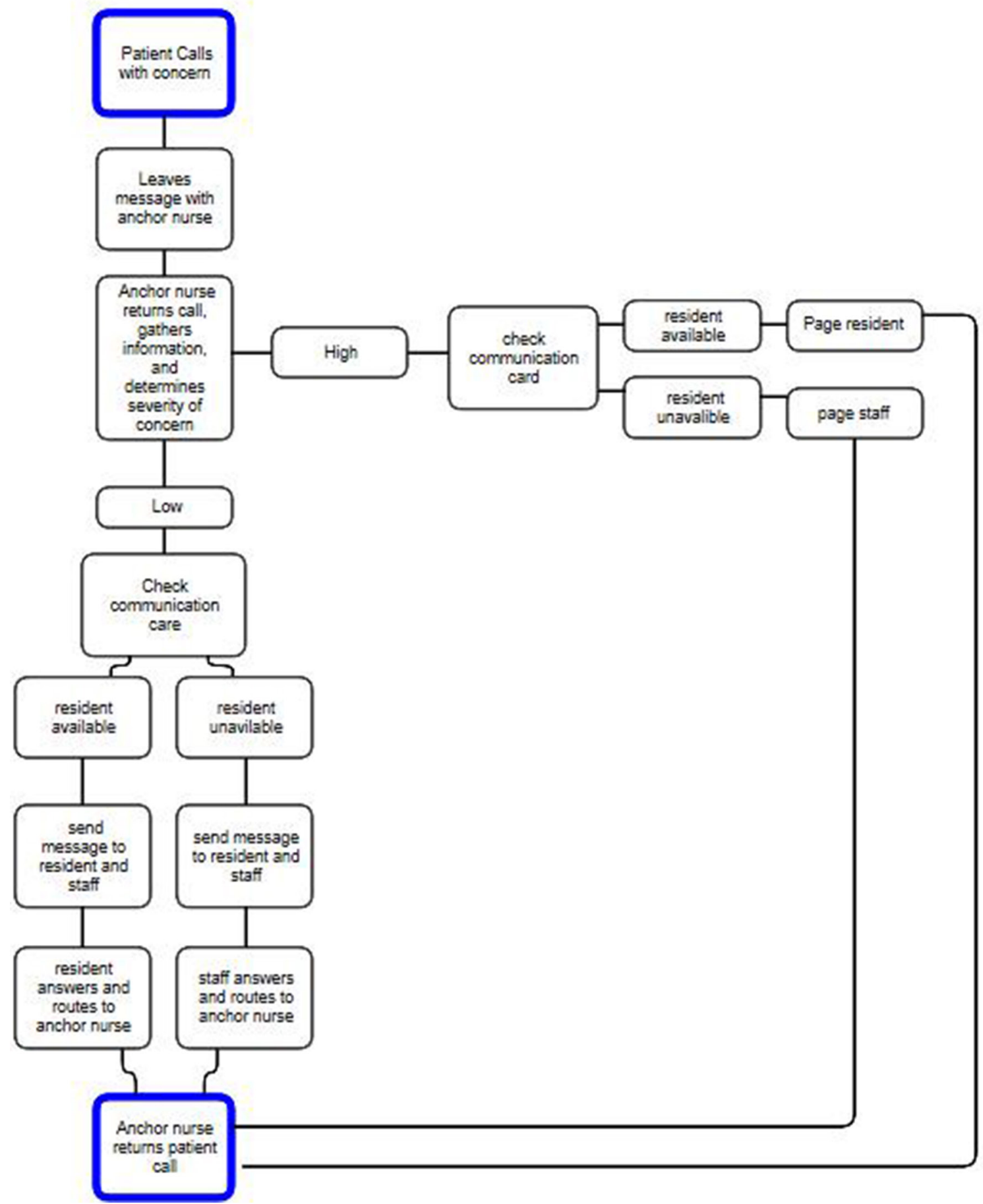

Figure 2 New communication algorithm and protocol.

communication. In addition, pages were sent to residents twice per week for a 6-month duration to remind them to check their inbox in the EMR .

\section{STRATEGY}

Our initial aim was to decrease open telephone encounters in resident clinic by $15 \%$. Given our initial success with plan, do, study, act (PDSA) cycle 1, we decided to continue our project aim and pursue a total of four PDSA cycles and extend our project timeline to 19 months to test sustainability.

PDSA cycle 1: Our initial intervention was to target specific individual residents and faculty with attributable variation as identified by our data set. These individuals had a consistently higher than average number of open telephone encounters that were not resolved. An email from programme leadership was sent to these individuals outlining the new clinic policy and need for improvement in patient-physician communication. This intervention 
achieved a decrease in open telephone encounters from 270 to 165 , a decrease of $38.9 \%$.

PDSA cycle 2: In order to continue education throughout the clinic care teams, the communication algorithm and process map were posted and made widely available. The policy re-emphasised all resident physicians to monitor and complete patient calls twice weekly between scheduled continuity clinic weeks and daily during scheduled continuity clinic weeks. The policy also outlined rotations that were exempt from the frequent monitoring, where the faculty preceptor would be in charge of completing the resident's inbox items. The process map gave a visual representation of the ideal communication algorithm. It started with the patient call and branched out into arms based on severity of concern. Further branches dealt with communication between the nurse, resident physician and faculty member and what to do if the physician(s) did not answer. Evaluations of residents completed by anchor nurses and faculty preceptors regarding inbox management with an emphasis on intercommunication skills, professionalism and systems of care were implemented. These new changes were disseminated in an educational session given to faculty and anchor nurses in the clinic by the clinic leadership and leaders of the quality improvement team. This intervention achieved an additional decrease in open telephone encounters to 132, an additional 20\% decrease.

PDSA cycle 3: Faculty preceptors were charged with disseminating the new policies and communication algorithm to resident physicians within their aligned care team. The policy identified the faculty preceptor as the ultimate party responsible for inbox management. Faculty preceptors were encouraged to allow resident autonomy in patient care when appropriate. In order to reinforce timely communication between the resident physician and their patients, twice weekly pages were sent out for 6 months to remind the resident physician to check their EMR inbox. After this intervention, there was a slight increase in total open telephone encounters. However, overall decline in open telephone encounters was still $40 \%$ from project initiation.

PDSA cycle 4: Our final test cycle was aimed at instilling a self-sustaining culture change in the resident continuity clinic by incorporating the clinic communication algorithm into orientation for both new anchor nurses and new residents. With no additional interventions, open telephone encounters still decreased by $40 \%$ overall after 11 months of PDSA cycle 4.

Total duration of all PDSA cycles was 19 months. These cycles yielded a sustained and acceptable improvement in practice standards for telephone communication.

\section{RESULTS}

Results were captured on a bimonthly basis for all 65 residents in the study. Data were collected from 15 November 2015 to 1 June 2017, as referenced in table 1. Of note, 65 total residents were evaluated throughout the study,

\begin{tabular}{ll}
\hline Table 1 & Open telephone encounters \\
\hline Date & Open telephone encounters \\
\hline 1 November 2015 & 270 \\
\hline 15 November 2015 & 228 \\
\hline 1 December 2015 & 219 \\
\hline 15 December 2015 & 218 \\
\hline 1 January 2016 & 132 \\
\hline 15 January 2016 & 150 \\
\hline 1 February 2016 & 165 \\
\hline 15 February 2016 & 132 \\
\hline 1 March 2016 & 146 \\
\hline 15 March 2016 & 147 \\
\hline 1 April 2016 & 132 \\
\hline 15 April 2016 & 108 \\
\hline 15 May 2016 & 98 \\
\hline 1 June 2016 & 151 \\
\hline 15 June 2016 & 140 \\
\hline 1 May 2016 & 163 \\
\hline 1 June 2017 & 160 \\
\hline
\end{tabular}

however, specific physicians were variable due to graduation and matriculation.

PDSA cycle 1 directed intervention to individuals with attributable risk. This resulted in lowering open telephone encounters from 270 to 165 , a decrease of $38.9 \%$.

PDSA cycle 2 from 1 February 2016 through 1 April 2016 resulted in a lowering of open telephone encounters from 165 to 132 , a $20 \%$ decrease.

PDSA cycle 3 from 1 April 2016 through 30 June 2016, showed in a small increase in open encounters compared with PDSA cycle 2, but still a dramatic improvement from our initial PDSA cycle (overall $40 \%$ decrease).

PDSA cycle 4 from 1 July 2016 through 1 June 2017 was to demonstrate sustainability. This final PDSA cycle demonstrated a consistent lowering of $40 \%$ from the beginning of our study.

Results from each PDSA cycle are graphically pictured in figure 3 .

\section{LESSONS AND LIMITATIONS}

The project aim was to improve open telephone encounters in a resident physician continuity clinic by utilising a multidisciplinary team and standardisation of a complex communication algorithm.

Our findings reflect that by creating a telephone process map, a complicated algorithm can be simplified to improve inbox open encounters in a busy, academic, internal medicine residency practice. We feel the primary intervention for initial success was the new process map, but we have been able to maintain success by changing the culture around inbox management through the combined interventions. 


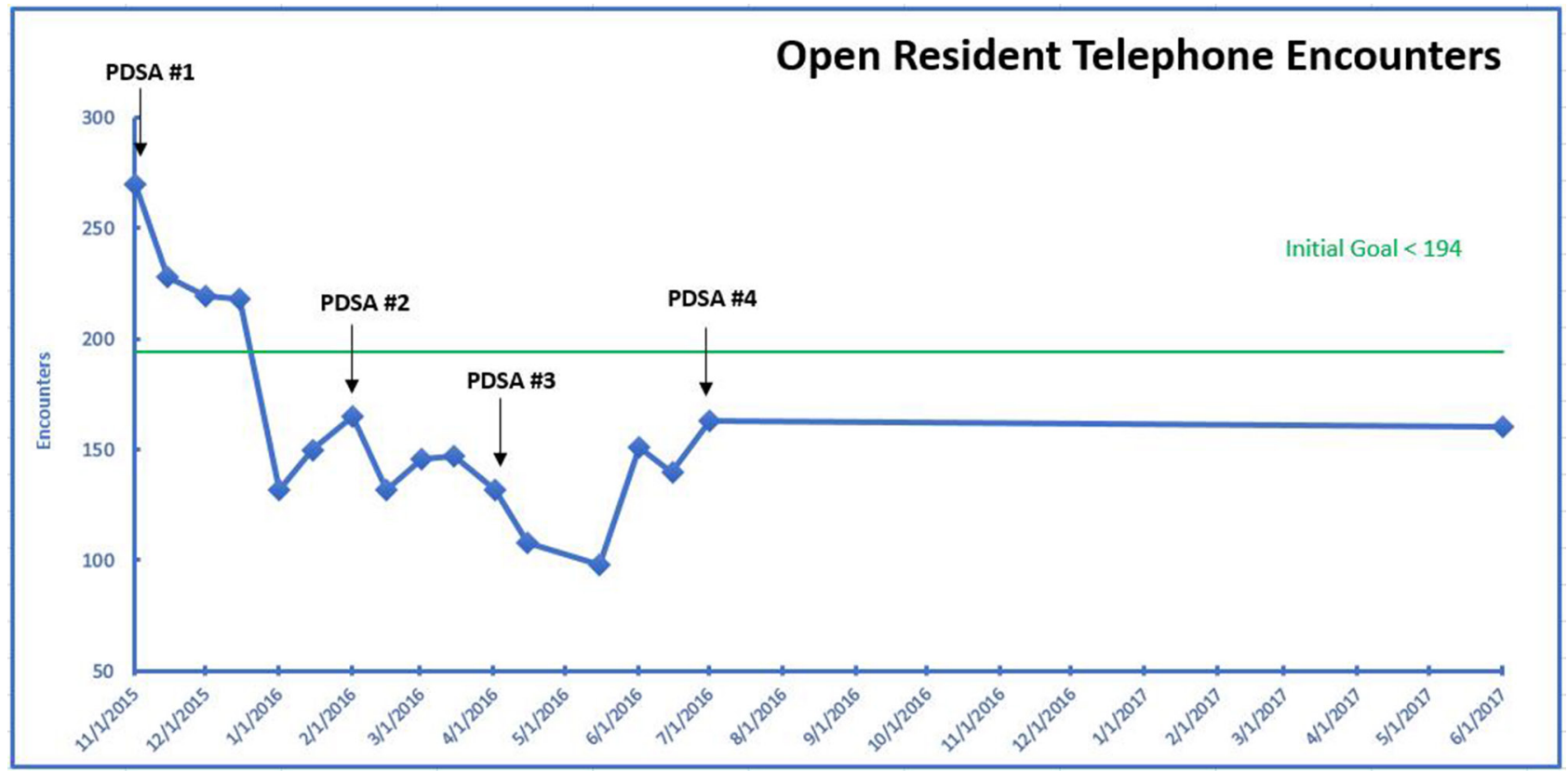

Figure 3 Open resident telephone encounters.

With no cost, our project was successful and sustainable at 19 months postintervention.

Our key limitation was the utility of the enterprise generated report as our primary measurement tool. This report has inherent barriers; mainly, it is a point-in-time measurement. As it is a point-in-time measurement, we cannot ensure accurately that it is a true reflection of care team communication and telephone response times. However, a strength is that it is an extremely precise data set that is consistent and institutionally derived. An additional limitation was our small, but consistent number of resident physician outliers. As these resident physician outliers remained, a future direction may look at the motivation of these residents and how to improve their individual involvement in multidisciplinary systems-based practice.

This quality improvement project highlighted many positive lessons. Specifically, the use of multiple PDSA cycles allowed for adaptability and reassessment throughout the project. Additionally, we learnt that defining clear roles of the care team can empower each individual to improve communication. Choosing a project that was important to both leadership and the care team, as well as developing a diverse focus group was essential to show sustainability as all key components were represented from the beginning. This project demonstrated that a low-cost intervention, grounded in simplification of a current process, could instigate culture change resulting in significant improvement.

\section{CONCLUSION}

Implementation of a standardised algorithm and communication tool through a quality improvement project led to positive outcomes for resident telephone communication. We were able to create a multidisciplinary culture change by putting forth clear expectations. This culture change is self-sustaining as we have implemented the algorithms and expectations into intern, faculty and nurse orientation. As the initial training was delivered in pre-existing meeting times, this intervention was at no added cost. Telephone management issues are similar for other primary care disciplines, and creation of this novel, low-cost algorithm and multidisciplinary practice management guide may be useful for family medicine, paediatrics and subspecialty clinicians alike. The ease of this project showed that a small, but well defined, quality improvement initiative can be effectively completed in a residency training programme and can be generalisable to others.

Contributors All authors equally contributed to the planning, conducting and reporting of the work.

Competing interests None declared.

Provenance and peer review Not commissioned; externally peer reviewed.

Open Access This is an Open Access article distributed in accordance with the Creative Commons Attribution Non Commercial (CC BY-NC 4.0) license, which permits others to distribute, remix, adapt, build upon this work non-commercially, and license their derivative works on different terms, provided the original work is properly cited and the use is non-commercial. See: http://creativecommons.org/ licenses/by-nc/4.0/

(c) Published by the BMJ Publishing Group Limited. For permission to use (where not already granted under a licence) please go to http://www.bmj.com/company/ products-services/rights-and-licensing/

\section{REFERENCES}

1. Reisman AB, Stevens DL, eds. Telephone medicine: a guide for the practicing physician. Philadelphia: American College of Physicians, 2002. 
2. Flannery MT, Moses GA, Cykert S, et al. Telephone management training in internal medicine residencies: a national survey of program directors. Acad Med 1995;70:1138-41.

3. Elnicki DM, Ogden P, Flannery M, et al. Telephone medicine for internists. J Gen Intern Med 2000;15:337-43.

4. Mueller PS, Barrier PA, Call TG, et al. Views of new internal medicine faculty of their preparedness and competence in physician-patient communication. BMC Med Educ 2006;6:30.

5. Delichatsios H, Callahan M, Charlson M. Outcomes of telephone medical care. J Gen Intern Med 1998;13:579-85.
6. Hannis MD, Elnicki DM, Morris DK, et al. Can you hold please? How internal medicine residents deal with patient telephone calls. Telephone Encounters Learning Initiative Group. Am J Med Sci 1994;308:349-52.

7. Reisman $\mathrm{AB}$, Brown KE. Preventing communication errors in telephone medicine: a case-based approach. J Gen Intern Med 2005;20:959-63.

8. Gandhi TK, Kachalia A, Thomas EJ, et al. Missed and delayed diagnoses in the ambulatory setting: a study of closed malpractice claims. Ann Intern Med 2006;145:488-96.

9. Katz HP, Kaltsounis D, Halloran L, et al. Patient safety and telephone medicine : some lessons from closed claim case review. J Gen Intern Med 2008;23:517-22. 